\title{
Identification of key candidate genes for colorectal cancer by bioinformatics analysis
}

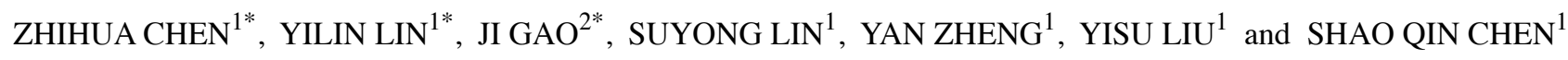 \\ ${ }^{1}$ Department of Gastrointestinal Surgery, The First Affiliated Hospital of Fujian Medical University; \\ ${ }^{2}$ School of Nursing, Fujian Medical University, Fuzhou, Fujian 350004, P.R. China
}

Received December 27, 2018; Accepted August 16, 2019

DOI: $10.3892 / \mathrm{ol} .2019 .10996$

\begin{abstract}
Colorectal cancer (CRC) is one of the most common cancers of the digestive tract. Although numerous studies have been conducted to elucidate the cause of CRC, the exact mechanism of CRC development remains to be determined. To identify candidate genes that may be involved in CRC development and progression, the microarray datasets GSE41657, GSE77953 and GSE113513 were downloaded from the Gene Expression Omnibus database. Gene Ontology and Kyoto Encyclopedia of Genes and Genomes were used for functional enrichment analysis of differentially expressed genes (DEGs). A protein-protein interaction network was constructed, and the hub genes were subjected to module analysis and identification using Search Tool for the Retrieval of Interacting Genes/Proteins and Cytoscape. A total of 142 DEGs were identified, with enriched functions and pathways in the 'cell cycle', 'cell proliferation', 'the mitotic cell cycle' and 'one-carbon metabolic process'. In addition, 10 hub genes were identified, and functional analysis indicated that these genes are mainly enriched in 'cell division', 'cell cycle' and functions associated with nucleotide binding processes. Survival analysis demonstrated that DNA topoisomerase II $\alpha$, cyclin-dependent kinase 1 and CDC28 protein kinase regulatory subunit 2 may be involved in cancer invasion or recurrence. The DEGs identified in the present study may help explain the molecular mechanisms of CRC development and progression.
\end{abstract}

Correspondence to: Professor Shao Qin Chen, Department of Gastrointestinal Surgery, The First Affiliated Hospital of Fujian Medical University, 20 Chazhong Road, Taijiang, Fuzhou, Fujian 350004, P.R. China

E-mail: chenshaoqln1613@163.com

${ }^{*}$ Contributed equally

Key words: colorectal cancer, differentially expressed genes, bioinformatics

\section{Introduction}

Colorectal cancer (CRC) ranks third (13.5\%) and second (9.5\%) among the incidence of malignancies worldwide in male and female patients, respectively, and is a serious hazard to human health (1). Previous studies have demonstrated that the molecular pathogenesis of CRC is mostly caused by genetic mutations $(2,3)$. Numerous studies over the past two decades have reported that genetic mutations are associated with the prognosis and treatment of CRC, and targeted therapies have been developed (4-7). The progression of CRC is usually accompanied by the activation of the $K R A S$ and $B R A F$ genes and the inhibition of the p53 tumour suppressor gene expression; mutations in these genes are associated with changes in the number and structure of chromosomes (8-10). However, $>15 \%$ of sporadic CRCs occur through completely different molecular pathogenesis. For example, serrated precancerous lesions usually manifest as a result of the methylation of the $\mathrm{CpG}$ locus and mutation of the gene (11).

The prognosis of CRC is poor due to a lack of effective diagnostic methods at an early stage. Therefore, an effective solution can only be provided for subsequent diagnosis and treatment by better understanding the gene expression of CRC during its occurrence and development and identifying the genes that may be involved in the occurrence and progression of cancer.

With the rapid development of science and technology, microarray technology has become increasingly accurate and has been widely used to explore changes in animal and plant gene expression (12-14). Microarray technology aids in the discovery of changes in gene expression during cancer development and progression (15-17). However, it can be difficult to obtain reliable results with single microarray analysis. The present study aimed to identify genetic changes in CRC in three mRNA microarray datasets from the Gene Expression Omnibus (GEO) database by obtaining differentially expressed genes (DEGs) between CRC and normal tissues. Gene Ontology (GO) and Kyoto Encyclopedia of Genes and Genomes (KEGG) were used for functional enrichment analysis, and a protein-protein interaction (PPI) network was used to analyse the associations between the DEGs. A total of 142 DEGs and 10 hub genes were identified, which may be candidate biomarkers of CRC. 


\section{Materials and methods}

Microarray data. GEO (http://www.ncbi.nlm.nih.gov/geo) (18) is a public functional genomic database. Three gene expression datasets (GSE41657, GSE77953, and GSE113513; analysed on Agilent-014850 Whole Human Genome Microarray 4x44K G4112F, Affymetrix Human Genome U133A Array, and Affymetrix Human Gene Expression Array platforms, respectively) were downloaded from GEO. The probes were converted to the corresponding gene symbols based on the annotation information on the platform. The GSE41657 dataset contained $25 \mathrm{CRC}$ and 12 adjacent normal tissue samples. The GSE77953 dataset contained $28 \mathrm{CRC}$ and 13 non-tumour samples. The GSE113513 dataset contained 14 CRC and 14 non-tumour samples.

Identification of DEGs. DEGs between CRC and non-tumour samples were screened using GEO2R (http://www.ncbi. nlm.nih.gov/geo/geo2r). GEO2R is an interactive web tool that compares two or more GEO datasets to identify DEGs across experimental conditions. Adjusted P-values (adj. P) and Benjamin and Hochberg false discovery rates were applied to balance between statistically significant findings and false-positive limits. Probe sets without corresponding gene symbols were removed, and genes with more than one probe set were averaged. Log [fold change(FC)] $>1$ and adj. P-value $<0.01$ were considered to indicate a statistically significant difference.

KEGG and GO enrichment analysis of DEGs. The Database for Annotation, Visualization and Integrated Discovery (DAVID; version 6.7; http://david.ncifcrf.gov) (19) is an online bioinformatics database that integrates biological data and analysis tools and provides gene annotation information and protein data. KEGG is a database resource for understanding advanced functions and biological systems from large-scale molecular data generated by high-throughput experimental techniques (20). GO is a major bioinformatics tool for annotating genes and analysing their biological processes (BPs), molecular functions (MFs) and cellular components (CCs) (21). To analyse the function of the DEGs, analysis was performed using DAVID. $\mathrm{P}<0.05$ was considered to indicate a statistically significant difference. The results based on the top ten BPs, MFs, CCs and KEGG were visualized.

PPI network construction and module analysis. A PPI network was predicted using the Search Tool for the Retrieval of Interacting Genes/Proteins (STRING; version 10.0; http://string-db.org) database (22). The PPI network of the DEGs was constructed, and interactions with a combined score $>0.4$ were considered statistically significant. Cytoscape (version 3.4.0) is an open source bioinformatics platform for visualizing molecular interaction networks (23). Cytoscape plug-in Molecular Complex Detection (MCODE; version 1.4.2) provides topology-based clustering for a network (24). Cytoscape was used to draw a PPI network, and MCODE was used to identify the most important modules in the network. The selection criteria were as follows: MCODE score $>5$, degree cut-off $=2$, node score cut-off $=0.2$, maximum depth $=100$, and $\mathrm{k}$ score $=2$. Subsequently, KEGG and GO analysis of the genes in this module was performed using DAVID.

Hub genes selection and analysis. The selection criterion for the hub genes was degree of connectivity $\geq 10$. The functions of the genes were identified using the GeneCards online analysis tool (https://www.genecards.org). The BP analysis and visualization of the hub genes were performed using the cBioPortal (http://www.cbioportal.org) online platform (25). The functions of the hub genes were identified using GO and KEGG analysis. Hierarchical clustering of the hub genes was constructed using the University of California Santa Cruz Cancer Genomics Browser (http://genome-cancer.ucsc. edu) (26). The overall survival analysis was performed using Kaplan-Meier curves in cBioPortal. The log-rank test was used to for statistical analysis. The expression profiles of DNA topoisomerase II $\alpha$ (TOP2A) and phosphoribosylaminoimidazole carboxylase (PAICS) were analysed using the Oncomine database (http://www.oncomine.com). The expression levels of genes in normal tissues and CRC tissues were also analysed using the Oncomine database (27).

\section{Results}

Determination of DEGs in CRC. DEGs were identified by standardized microarray results (GSE41657, GSE77953 and GSE113513). A total of 142 genes overlapped in the three datasets, as demonstrated in the Venn diagram (Fig. 1A).

PPI network and module analysis. A PPI network of the 142 identified DEGs was constructed (Fig. 1B). A significantly enriched module was obtained using Cytoscape software (Fig. 1C).

GO and KEGG enrichment analysis of the DEGs. DAVID was used for the functional enrichment analysis of DEGs. The results indicated that the DEGs were mainly enriched in 'cell proliferation', 'G2/M transition of mitotic cell cycle' and 'one-carbon metabolism' BPs (Fig. 2A); 'cytoplasm', 'cytosol' and 'extracellular exosome' cellular components (CCs) (Fig. 2B); and 'protein binding' and 'ATP binding' molecular functions (MFs) (Fig. 2C). KEGG pathway analysis revealed strong enrichment in 'biosynthesis of antibiotics', 'purine metabolism', 'pancreatic secretion' and 'mineral absorption' (Fig. 2D).

The functional results of the genes in the most significant module indicated that these genes were mainly enriched in processes associated with cell cycle progression, ATP binding, nucleotide binding, the p53 signalling pathway and oocyte meiosis (Table I).

Hub genes screening and analysis. Genes with a degree of connectivity $\geq 10$ were identified as the hub genes. The names, abbreviations and functions of these genes are presented in Table II. The cBioPortal online platform was used to construct a hub gene network (Fig. 3A), and functional enrichment analysis of DEGs was performed using DAVID (Table III). The heat map constructed using the UCSC Cancer Genomics Browser indicated that the hub genes may be used to distinguish CRC tissue samples from normal intestinal cancer tissue samples (Fig. 3B). Overall and progression-free survival 
A

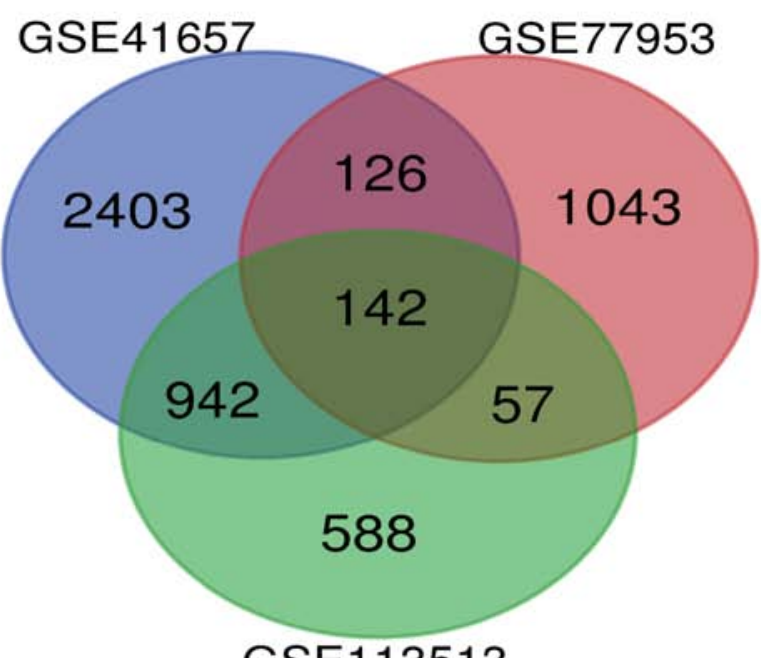

C

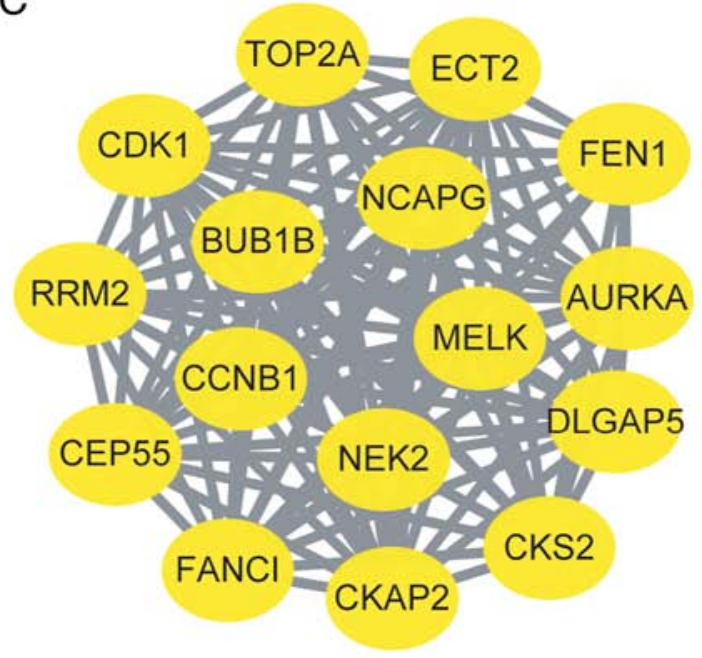

B

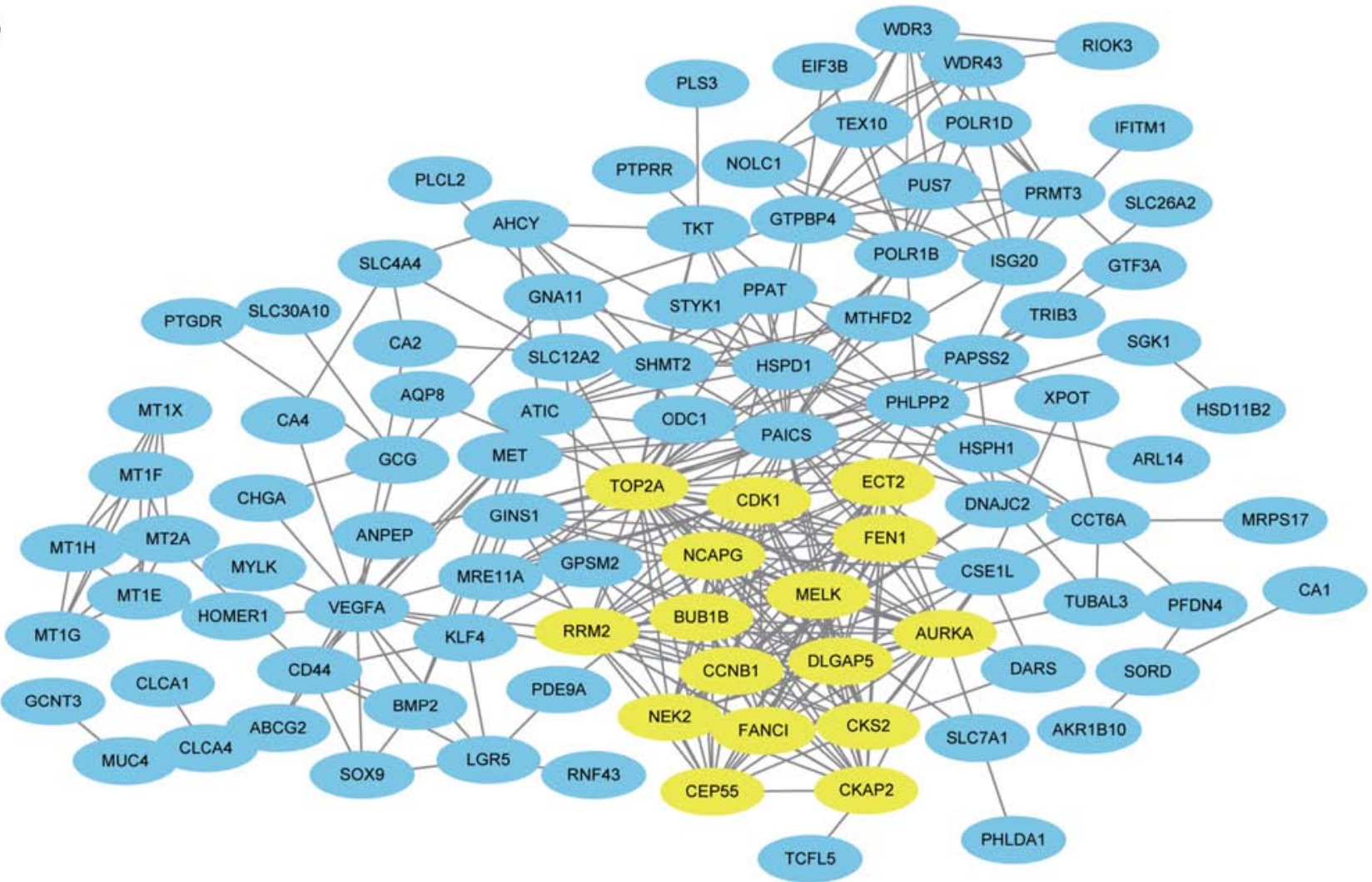

Figure 1. DEGs between CRC and non-tumour tissues. (A) Venn diagram of the DEGs in the three data sets (GSE41657, GSE77953, and GSE113513). Log fold change $>1$ and adjusted P-value $<0.01$ were the conditions for screening DEGs. A total of 142 DEGs were screened. (B) PPI network of 142 DEGs. Yellow indicates the densest module. (C) The relationships among the most important module genes in the PPI network. DEGs, differentially expressed genes; CRC, colorectal cancer; PPI, protein-protein interaction.

based on alterations in the hub genes was subsequently analysed using Kaplan-Meier curves. Alterations in TOP2A, cyclin-dependent kinase 1 (CDK1) and CDC28 protein kinase regulatory subunit 2 (CKS2) in patients with CRC were associated with a poor overall survival rate (Fig. 4A). However, patients with alterations in TOP2A and CKS2 did not exhibit significant differences in progression-free survival (Fig. 4B).

Among these genes, TOP2A exhibited a node degree of 34, and PAICS exhibited the highest degree of connectivity among the hub genes (34 and 26, respectively). These two genes may serve important roles in the occurrence or development of CRC. Based on the survival analysis, alterations in PAICS exhibited no statistically significant differences in overall and progression-free survival in patients with CRC (overall survival $\mathrm{P}=0.986$; progression-free survival $\mathrm{P}=0.918$; Fig. 4). Overall survival rates were significantly different for patients with TOP2A alterations, but progression-free survival was not significantly different (overall survival $\mathrm{P}=0.002$; progression-free survival $\mathrm{P}=0.703$; Fig. 4 ). The expression profiles of TOP2A and PAICS in human tissues were 
A

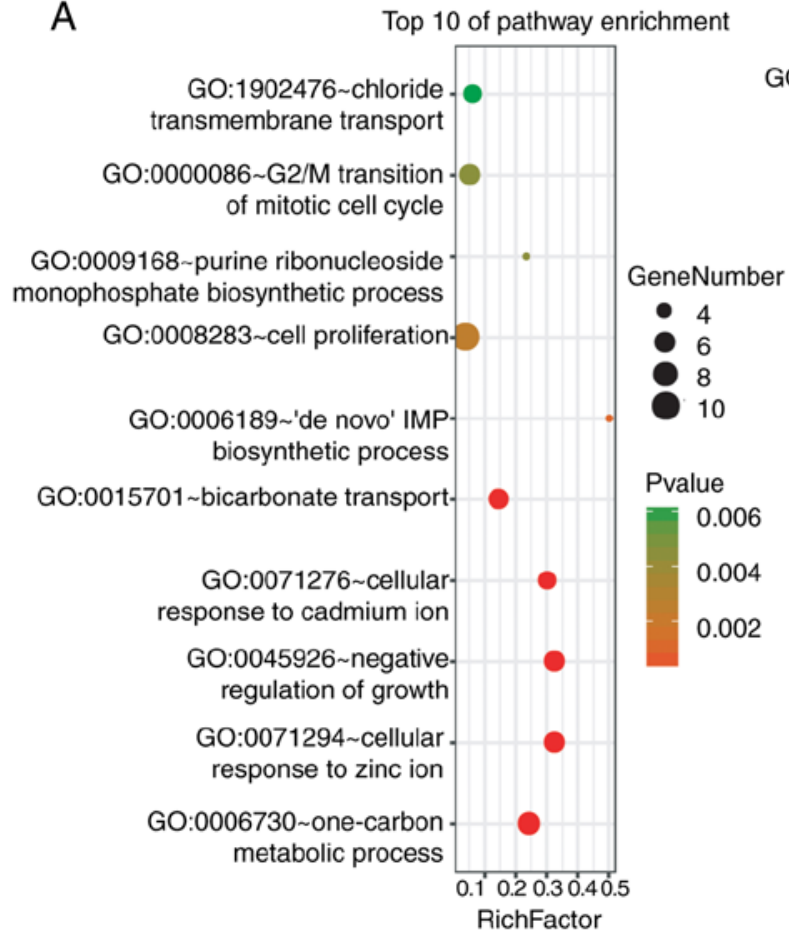

C

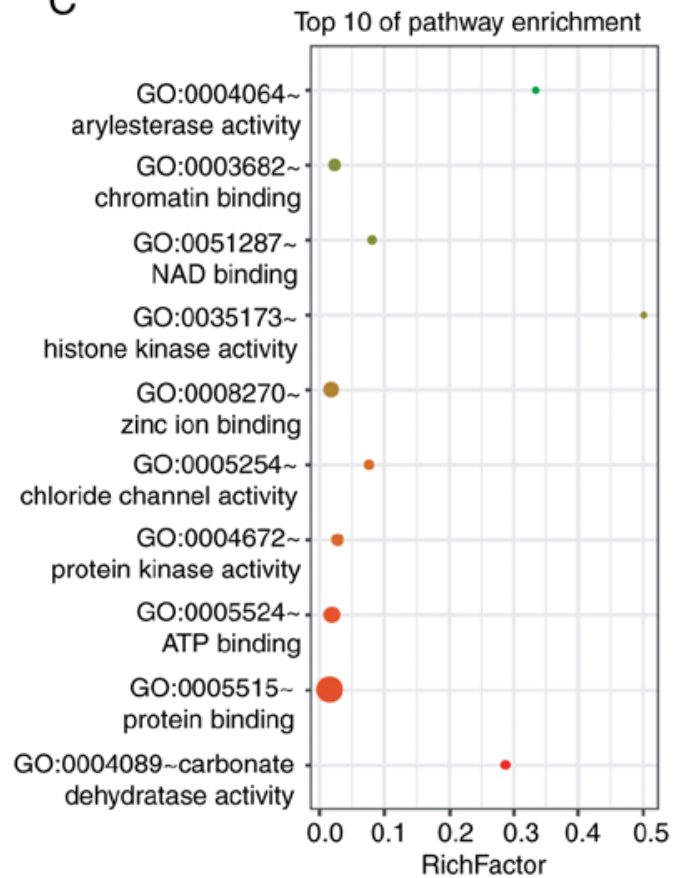

B

Top 10 of pathway enrichment

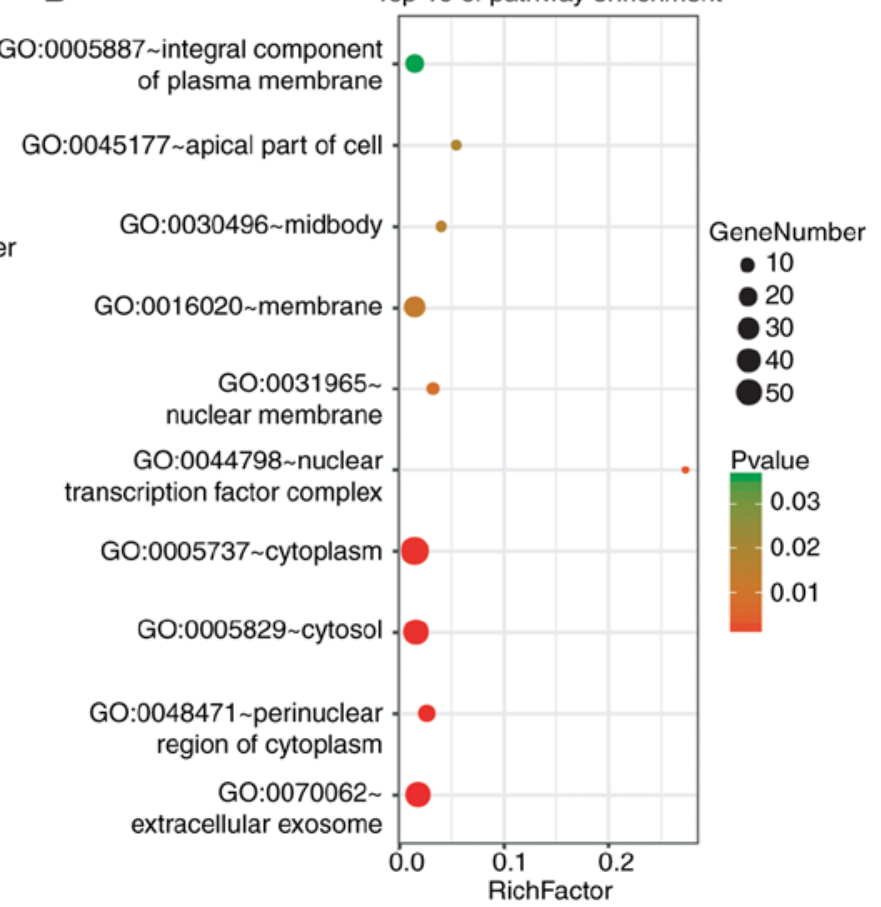

D

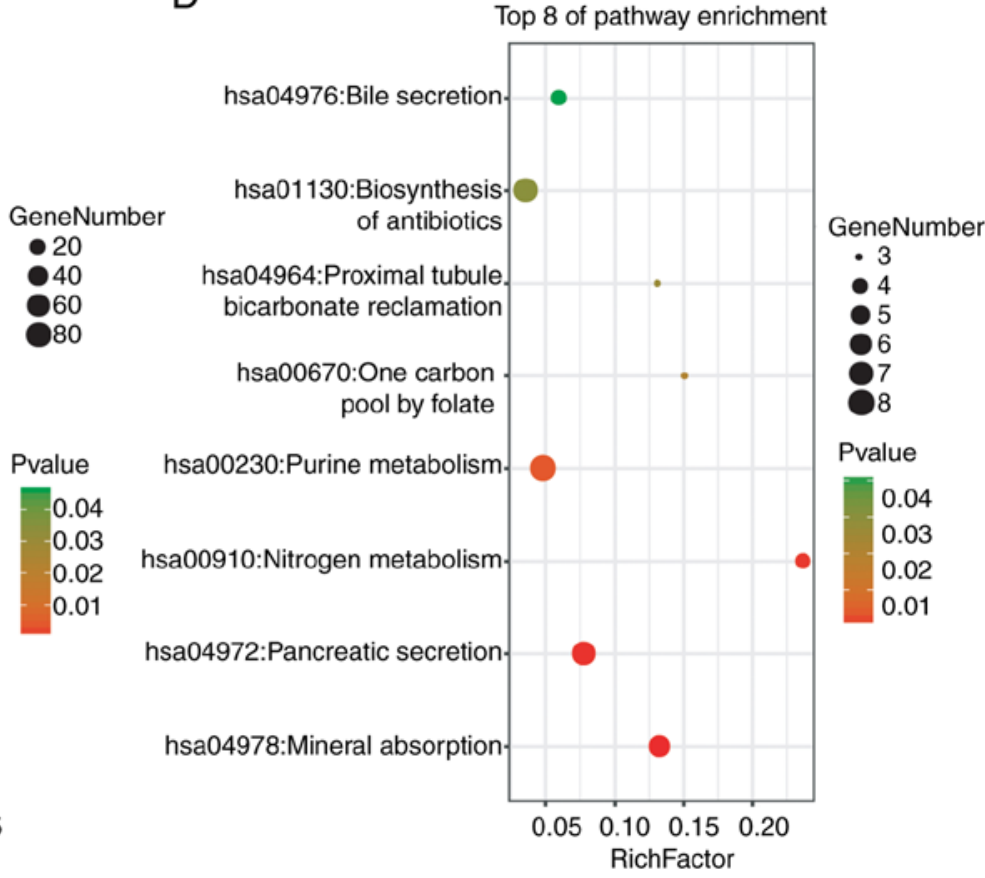

Figure 2. GO and KEGG pathway enrichment analysis of the DEGs. (A) The enrichment of biological processes. (B) The enrichment of cellular components. (C) The enrichment of molecular functions. (D) The enrichment analysis of the KEGG pathways. $\mathrm{P}<0.05$ was considered to indicate a statistically significant difference. GO, gene ontology; KEGG, Kyoto Encyclopedia of Genes and Genomes; DEGs, differentially expressed genes.

analysed using the Oncomine database; TOP2A and PAICS mRNA was upregulated in various cancer tissues compared with normal tissues (Fig. 5A and B). Further analysis revealed significant increases in the expression of TOP2A and PAICS in CRC (Fig. 5C and D).

\section{Discussion}

CRC is a common malignant tumour of the digestive tract, and its mortality rate $(9.2 \%)$ ranks second among all types of cancer (1). The main causes of CRC include dietary and environmental factors, as well as genetic mutations (3). The simultaneous methylation of the $\mathrm{CpG}$ site and mutation of the BRAF gene are also important factors in the development and progression of CRC (11). Failure to detect CRC early may be one of the reasons for poor prognosis in patients. Therefore, there is an urgent need for efficient diagnostic and therapeutic methods.

In the present study, DEGs between CRC and non-cancerous tissues were obtained from three mRNA microarray datasets. 
Table I. GO and KEGG pathway enrichment analysis of DEGs in the most significant module.

\begin{tabular}{llrr}
\hline Term & \multicolumn{1}{c}{ Description } & No. of genes & P-value \\
\hline GO:0007049 & Cell cycle & 11 & $8.42 \times 10^{-10}$ \\
GO:0000279 & M phase & 9 & $6.24 \times 10^{-10}$ \\
GO:0022403 & Cell cycle phase & 9 & $3.84 \times 10^{-9}$ \\
GO:0022402 & Cell cycle process & 9 & $4.38 \times 10^{-8}$ \\
GO:0043228 & Non-membrane-bounded organelle & 11 & $1.83 \times 10^{-5}$ \\
GO:0015630 & Microtubule cytoskeleton & 9 & $1.17 \times 10^{-8}$ \\
GO:0044430 & Cytoskeletal part & 9 & $8.45 \times 10^{-7}$ \\
GO:0005524 & ATP binding & 6 & $2.91 \times 10^{-3}$ \\
GO:0032559 & Adenyl ribonucleotide binding & 6 & $3.10 \times 10^{-3}$ \\
GO:0030554 & Adenyl nucleotide binding & 6 & $3.91 \times 10^{-3}$ \\
hsa04115 & p53 signalling pathway & 3 & $1.72 \times 10^{-3}$ \\
hsa04114 & Oocyte meiosis & 3 & $4.44 \times 10^{-3}$ \\
hsa04110 & Cell cycle & 3 & $5.71 \times 10^{-3}$ \\
\hline
\end{tabular}

GO, Gene Ontology; KEGG, Kyoto Encyclopedia of Genes and Genomes.

Table II. Functional roles of the 10 hub genes with degree of connectivity $\geq 10$.

\begin{tabular}{|c|c|c|c|}
\hline No. & Gene symbol & Full name & Function \\
\hline 1 & TOP2A & DNA Topoisomerase II $\alpha$ & $\begin{array}{l}\text { Target for anticancer agents; mutations are associated with drug } \\
\text { resistance }\end{array}$ \\
\hline 2 & PAICS & $\begin{array}{l}\text { Phosphoribosylaminoimidazole } \\
\text { carboxylase }\end{array}$ & Involved in de novo synthesis of purine nucleotides \\
\hline 3 & CDK1 & Cyclin-dependent kinase 1 & $\begin{array}{l}\text { Regulates cell cycle progression, apoptosis and carcinogenesis } \\
\text { of tumour cells }\end{array}$ \\
\hline 4 & CKS2 & $\begin{array}{l}\text { CDC28 protein kinase regulatory } \\
\text { subunit } 2\end{array}$ & $\begin{array}{l}\text { Binds to the catalytic subunit of cyclin-dependent kinases; } \\
\text { essential for their biological function }\end{array}$ \\
\hline 5 & CKAP2 & Cytoskeleton associated protein 2 & $\begin{array}{l}\text { Possesses microtubule stabilizing properties; involved in } \\
\text { regulating aneuploidy, cell cycle and cell death in a p53/TP53- } \\
\text { dependent manner }\end{array}$ \\
\hline 6 & CEP55 & Centrosomal protein 55 & Promotes the proliferation of lung, breast and thyroid cancer \\
\hline 7 & VEGFA & $\begin{array}{l}\text { Vascular endothelial growth } \\
\text { factor A }\end{array}$ & $\begin{array}{l}\text { Induces endothelial cell proliferation, promotes cell migration, } \\
\text { inhibits apoptosis and induces blood vessel permeabilization }\end{array}$ \\
\hline 8 & PHLPP2 & $\begin{array}{l}\text { PH domain and leucine rich repeat } \\
\text { protein phosphatase } 2\end{array}$ & Regulates Akt and PKC signalling. \\
\hline 9 & RRM2 & $\begin{array}{l}\text { Ribonucleotide reductase M2 } \\
\text { polypeptide }\end{array}$ & $\begin{array}{l}\text { Catalyses the biosynthesis of deoxyribonucleotides; inhibits } \\
\text { Wnt signalling }\end{array}$ \\
\hline 10 & NEK2 & $\begin{array}{l}\text { NIMA (never in mitosis gene a)- } \\
\text { related kinase } 2\end{array}$ & Involved in the regulation of mitosis \\
\hline
\end{tabular}

The functions of the DEGs were identified by GO and KEGG enrichment analysis. The results demonstrated that the DEGs were mainly enriched in the cell cycle, proliferation, mitotic cell cycle and carbon metabolism. Previous studies have reported that dysregulation of the cell cycle and the mitotic cell cycle serves an important role in the development and progression of CRC (28-31). In a study by Chamberlain et al (32), functional $\mathrm{B}$ vitamin was used to assess the association of carbon metabolism with $\mathrm{CRC}$, and a similar association between total B vitamin status and CRC risk was identified. Ducker (33) et al also revealed that the single-carbon metabolism of the cytosol can support tumourigenesis. These findings are consistent with the results of the present study. GO cluster and KEGG analysis in the present study also revealed that the changes in the most important module were mainly enriched in processes associated with cell cycle progression, ATP and 
Table III. GO and KEGG pathway enrichment analysis of hub genes.

\begin{tabular}{llcc}
\hline Term & \multicolumn{1}{c}{ Description } & No. of genes & P-value \\
\hline GO:0007049 & Cell cycle & 5 & $6.24 \times 10^{-4}$ \\
GO:0051301 & Cell division & 4 & $5.30 \times 10^{-4}$ \\
GO:0000279 & M phase & 4 & $7.29 \times 10^{-4}$ \\
GO:0015630 & Microtubule cytoskeleton & 5 & $1.06 \times 10^{-4}$ \\
GO:0044430 & Cytoskeletal part & 5 & $8.92 \times 10^{-4}$ \\
GO:0005856 & Cytoskeleton & 5 & $3.63 \times 10^{-3}$ \\
GO:0005524 & ATP binding & 4 & $3.62 \times 10^{-2}$ \\
GO:0032559 & Adenyl ribonucleotide binding & 4 & $3.75 \times 10^{-2}$ \\
GO:0030554 & Adenyl nucleotide binding & 4 & $4.29 \times 10^{-2}$ \\
hsa04115 & p53 signalling pathway & 2 & $3.96 \times 10^{-2}$ \\
\hline
\end{tabular}

GO, Gene Ontology; KEGG, Kyoto Encyclopedia of Genes and Genomes.

A

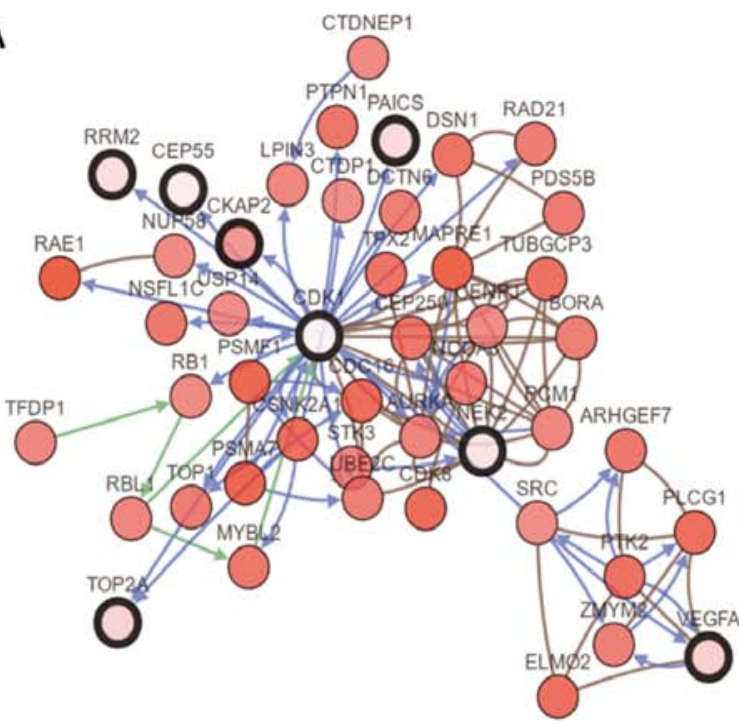

B

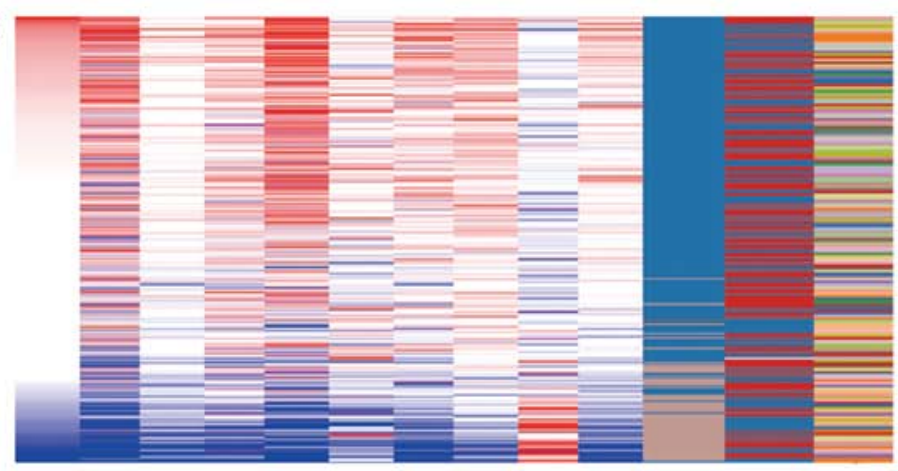

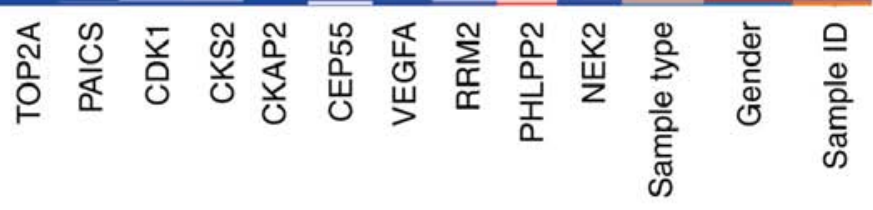

Figure 3. Interaction network of hub genes and hierarchical cluster analysis. (A) Analysis of the hub genes and their co-expressed genes using cBioPortal. (B) Hierarchical clustering of the hub genes. In the sample type column, brown samples are non-cancerous tissue samples, and blue samples are CRC tissue samples. In the gene expression columns, red indicates upregulated expression and blue indicates downregulated expression. CRC, colorectal cancer.

nucleotide binding and in KEGG pathways associated with the cell cycle, progesterone-mediated oocyte maturation and oocyte cell meiosis.

A total of 10 DEGs were selected as the hub genes. Among them, TOP2A and PAICS were the top two nodes with the highest degree of connectivity. TOP2A is involved in DNA replication, transcription and chromosome segregation, and is essential for tumorigenesis and cancer development (34). TOP2A has been demonstrated to be associated with chemoresistance and tumour recurrence and has been recognized as a target for anticancer drugs $(35,36)$. In addition, TOP2A is highly expressed in lung, colon and breast cancer, involved in the inhibition of apoptosis, proliferation and chemoresistance of CRC and may be considered an important biomarker for the diagnosis, prognosis and treatment of tumours (37-39). In the present study, the PPI network indicated that TOP2A interacted directly with CDK1, ribonucleoside-diphosphate reductase subunit $\mathrm{M} 2(\mathrm{RRM} 2)$ and CKS2, indicating a key role for TOP2A in CRC. PAICS is involved in purine nucleotide synthesis; a previous study has demonstrated that high expression of PAICS in lung and prostate cancer is associated with an altered metabolic state of cells, apoptosis inhibition in cancer cells and enhanced cancer cell invasion (40). In addition, PAICS has been reported to promote tumourigenesis and progression in breast and bladder cancer $(41,42)$. The results of the analysis in the Oncomine database in the present study demonstrated that TOP2A and PAICS expression was significantly upregulated in CRC compared with that in normal tissues; however, to the best of our knowledge, there are currently no studies focusing on the association between PAICS expression and CRC. The association between TOP2A and PAICS and overall and disease-free survival was also analysed; changes in TOP2A were significantly associated with overall survival but were independent of disease-free survival. Changes in PAICS exhibited a reduction in overall and disease-free survival; however, these observations were 


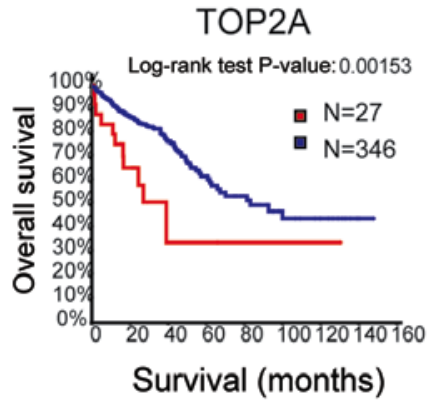

B

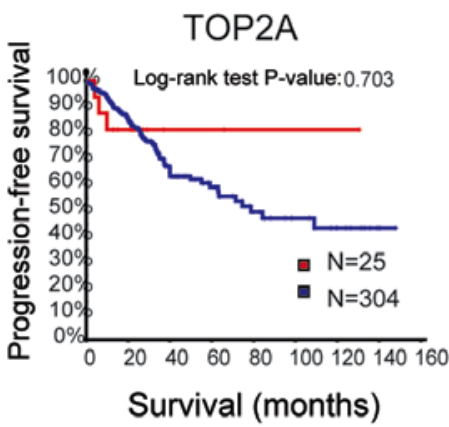

PAICS

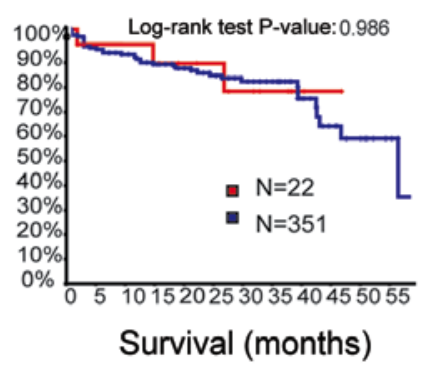

CDK1

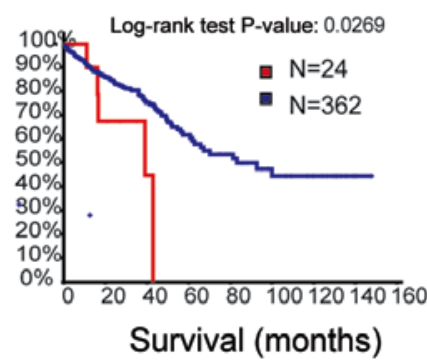

CKS2

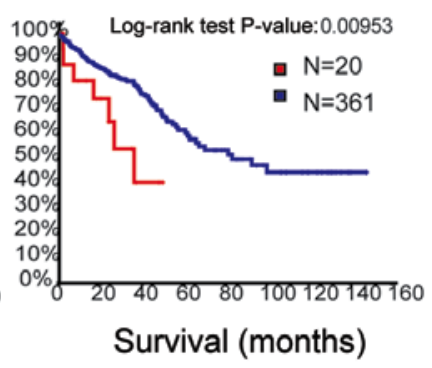

$\square$ Cases with alterations in query genes

a Cases without alterations in query genes

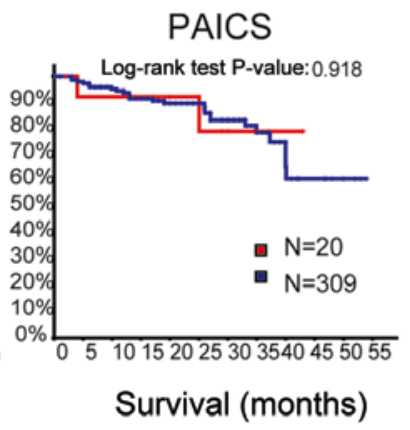

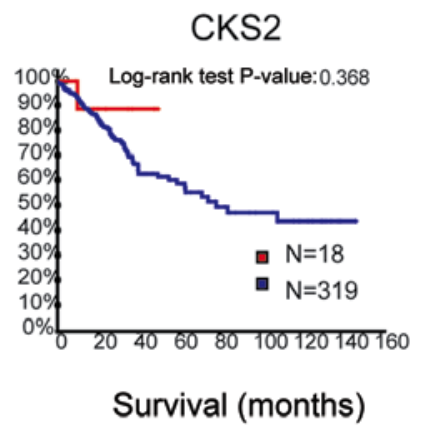

Figure 4. Analysis of overall survival and progression-free survival of four hub genes. (A) Overall survival and (B) progression-free survival analyses based on alterations in the hub genes were performed using the cBioPortal online platform. $\mathrm{P}<0.05$ was considered to indicate a statistically significant difference. TOP2A, DNA topoisomerase II $\alpha$; PAICS, phosphoribosylaminoimidazole carboxylase; CDK1, cyclin-dependent kinase 1; CKS2, CDC28 protein kinase regulatory subunit 2 .

not statistically significant. These results may require further research for verification.

CDK1 is a non-redundant cyclin-dependent kinase that serves an important role in mitosis $(43,44)$. Perturbations in chromosomal stability and aspects of $\mathrm{S}$ phase and G2/M control mediated by $\mathrm{CDK} 2$ and CDK1 are pivotal tumorigenic events (45). A previous study has demonstrated that CDK1 is required for the survival of cells overexpressing MYC, and CDK1 has a therapeutic effect in the treatment of human malignancies that overexpress MYC (46). The combination of CKS2 and CDK is essential for promoting cancer cell metastasis in diseases such as colon cancer $(47,48)$. Vascular endothelial growth factor A (VEGFA) is a class of cytokines called antigen growth factors that stimulate the formation of new blood vessels; tumour angiogenesis is primarily dependent on VEGFA-driven responses (49). Anti-VEGF-A treatment can reduce Treg proliferation in CRC patients, and VEGFA inhibitors have been used to treat CRC $(50,51)$. In the present study revealed, the expression levels of CDK11, CSK2 and VEGFA in CRC were analysed; the results revealed high expression of these genes in CRC, which was consistent with previous studies. In addition, high expression levels of CDK1 and CKS2 were associated with poor survival.

CKAP2 is a spindle-associated protein that is degraded during mitosis $(52,53)$. Upregulation of CEP55 promotes the metastasis of several cancers, such as lung adenocarcinoma, breast and anaplastic thyroid cancer (54-56). RRM2, also known as the ribonucleotide reductase small subunit, promotes cyclin $\mathrm{F}$ degradation to maintain a balanced dNTP pool and genomic stability (57). PHLPP2 is a protein phosphatase involved in the regulation of $\mathrm{Akt}$ and PKC signalling $(58,59)$. Previous studies have reported that microRNA-224 promotes the proliferation and tumour growth of human CRC cells by inhibiting PHLPP1 and PHLPP2 (60) and acts as a tumour suppressor for lung and breast cancer $(61,62)$. The results of the present study also demonstrated that it is downregulated in CRC tissues. NEK2 is a serine/threonine kinase that is a key regulator of mitosis in cells (63). Upregulation of NEK2 is associated with the development of cancer, such as breast (64), ovarian (65) and colorectal $(66,67)$ cancer. Overexpression of NEK2 promotes the invasion and metastasis of tumour cells and is considered a potential biomarker in nasopharyngeal carcinoma and liver cancer $(68,69)$. The results of the present study indicated that the aforementioned hub genes may be distinguishing factors between CRC and non-tumour tissue samples and candidate tumour biomarkers. In addition, alterations in TOP2A, CDK1 and CKS2 are associated with poor survival, suggesting that these genes may serve an important role in the development, progression or recurrence of cancer. 
A

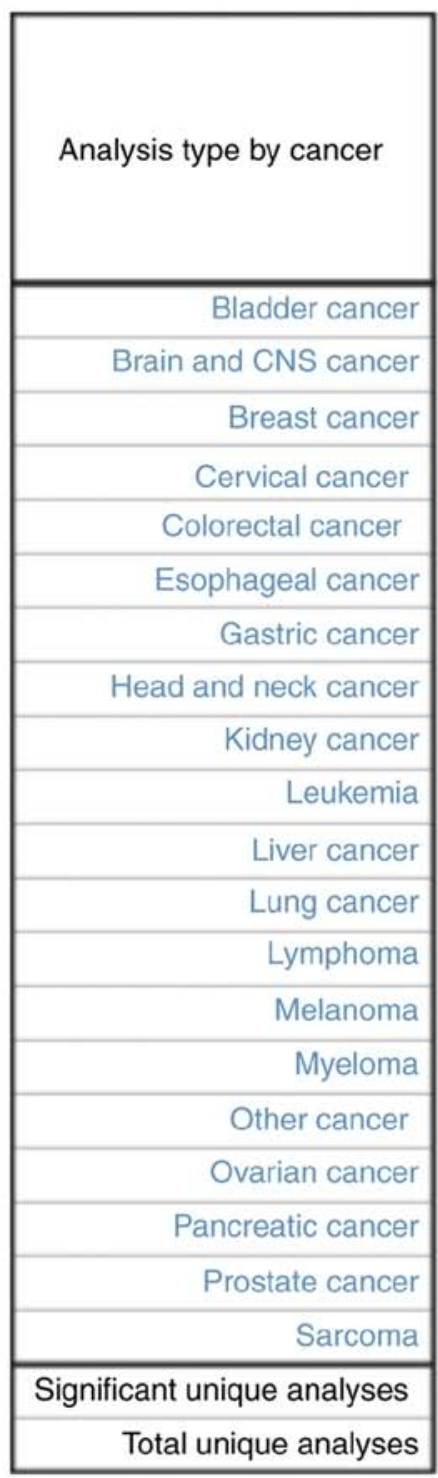

$\begin{array}{llllll}1 & 5 & 10 & 10 & 5 & 1\end{array}$

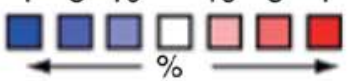

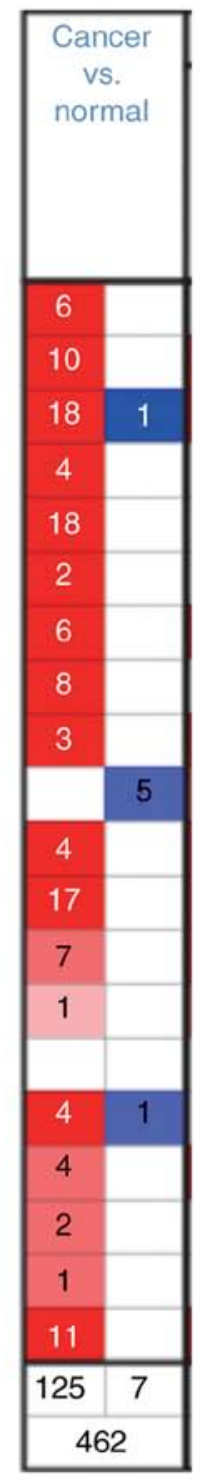

PAICS

$\begin{array}{llllll}1 & 5 & 10 & 10 & 5 & 1\end{array}$

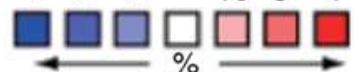

C

\begin{tabular}{|c|c|c|c|c|c|c|}
\hline Median rank & $p$-Value & Gene & & & & \\
\hline \multirow[t]{2}{*}{398.0} & $3.74 \mathrm{E}-5$ & TOP2A & & & & \\
\hline & & & 1 & 2 & 3 & 4 \\
\hline
\end{tabular}

D

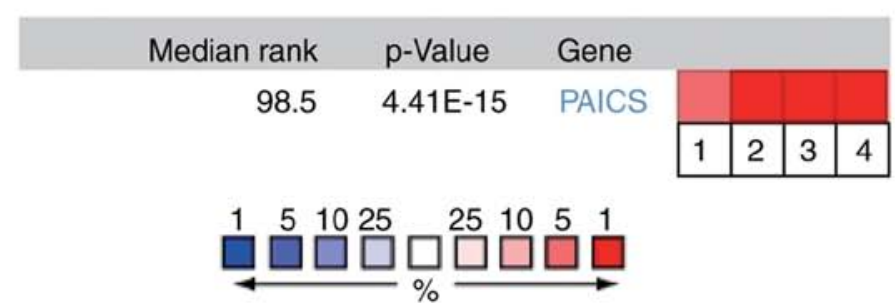

Analysis type by cancer

Bladder cancer

Brain and CNS cancer

Breast cancer

Cervical cancer

Colorectal cancer

Esophageal cancer

Gastric cancer

Head and neck cancer

Kidney cancer

Leukemia

Liver cancer

Lung cancer

Lymphoma

Melanoma

Myeloma

Other cancer

Ovarian cancer

Pancreatic cancer

Prostate cancer

Sarcoma

Significant unique analyses

Total unique analyses

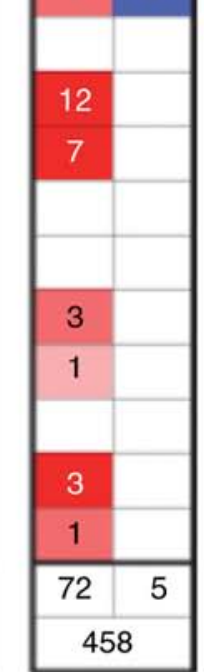

1. $P$-value $=1.41 \mathrm{E}-10$ fold-change $=3.777$

2. $P$-value $=9.10 E-8$ fold-change $=4.214$

3.P-value $=7.49 E-5$ fold-change $=3.750$

4. $P$-value $=7.75 E-9$ fold-change $=2.568$

1. $P$-value $=8.80 E-13$ fold - change $=3.428$

2. $P$-value $=2.41 E-28$ fold-change $=3.198$

3. $P$-value $=8.81 \mathrm{E}-15$ fold - change $=2.373$

4. $P$-value $=1.40 E-25$ fold-change $=3.202$
Cancer

vs.

normal

3

7

5

1

17

2

3

5

1

$\begin{array}{ll}1 & 4\end{array}$

Figure 5. Expression of TOP2A and PAICS in cancer and normal tissues. (A and B) The Oncomine online analysis platform was used to analyse the expression of (A) TOP2A and (B) PAICS in human cancer and normal tissues. (C) Heat map of TOP2A expression in clinical CRC and normal tissues. 1. Colorectal Carcinoma vs. Normal. Hong Colorectal. 2. Rectosigmoid Adenocarcinoma vs. Normal. Kaiser Colon. 3. Colon Adenocarcinoma vs. Normal. Notterman Colon. 4. Colorectal Carcinoma vs. Normal, Skrzypczak Colorectal. (D) Heat map of PAICS expression in clinical CRC tissues and normal tissues. Caecum Adenocarcinoma, Colon Adenocarcinoma, Colon Mucinous Adenocarcinoma and Rectal Adenocarcinoma vs. Normal. TCGA Colorectal. TOP2A, DNA topoisomerase II $\alpha$; PAICS, phosphoribosylaminoimidazole carboxylase; $\mathrm{CRC}$, colorectal cancer. 
In summary, the aim of the present study was to identify DEGs that may be involved in the development or progression of CRC. The results demonstrated that TOP2A and CDK1 may be involved in the survival prognosis of patients with CRC. The present study also revealed that these genes may be biomarkers for the diagnosis of CRC. However, the present study had limitations, and other databases such as The Cancer Genome Atlas, as well as in vivo and in vitro experiments may be needed to clarify the biological functions of these genes in CRC.

\section{Acknowledgements}

Not applicable.

\section{Funding}

The present study was supported by the Fujian Provincial Key Medical Specialist Construction Project (grant no. 2016-SLCZD), the Training Project of Young Talents in the Health System of Fujian Province (grant no. 2016-ZQN-45) and the Fujian Medical University Start up Fund for Scientific Research (grant no. 2017-XQ1061).

\section{Availability of data and materials}

The datasets used and analysed during the present study are available from the corresponding author on reasonable request.

\section{Authors' contributions}

SQC and ZHC designed the study and obtained funding YLL drafted the manuscript and supervised the study. YLL participates in the design of the study, obtaining data and writing manuscripts. YZ, YSL, JG and SYL collected and analysed the data. All authors read and approved the final manuscript.

\section{Ethics approval and consent to participate}

Not applicable.

\section{Patient consent for publication}

Not applicable.

\section{Competing interests}

The authors declare that they have no competing interests.

\section{References}

1. Bray F, Ferlay J, Soerjomataram I, Siegel RL, Torre LA and Jemal A: Global cancer statistics 2018: GLOBOCAN estimates of incidence and mortality worldwide for 36 cancers in 185 countries. CA Cancer J Clin 68: 394-424, 2018.

2. Van Cutsem E, Lenz HJ, Kohne CH, Heinemann V, Tejpar S, Melezinek I, Beier F, Stroh C, Rougier P, van Krieken JH and Ciardiello F: Fluorouracil, leucovorin, and irinotecan plus cetuximab treatment and RAS mutations in colorectal cancer. J Clin Oncol 33: 692-700, 2015.

3. Tong K,Pellón-Cárdenas O, Sirihorachai VR, WarderBN, KothariOA, Perekatt AO, Fokas EE, Fullem RL, Zhou A, Thackray JK, et al: Degree of tissue differentiation dictates susceptibility to BRAF-driven colorectal cancer. Cell Rep 21: 3833-3845, 2017.
4. Sanz-Garcia E, Argiles G, Elez E and Tabernero J: BRAF mutant colorectal cancer: Prognosis, treatment, and new perspectives. Ann Oncol 28: 2648-2657, 2017.

5. 5.Corcoran RB, André T, Atreya CE, Schellens JHM, Yoshino T, Bendell JC, Hollebecque A, McRee AJ, Siena S, Middleton G, et al: Combined BRAF, EGFR, and MEK inhibition in patients with BRAF(V600E)-mutant colorectal cancer. Cancer Discov 8: 428-443, 2018.

6. Hamzehzadeh L, Khadangi F, Ghayoor Karimiani E, Pasdar A and Kerachian MA: Common KRAS and NRAS gene mutations in sporadic colorectal cancer in Northeastern Iranian patients. Curr Probl Cancer 42: 572-581, 2018.

7. Reggiani Bonetti L, Barresi V, Bettelli S, Caprera C, Manfredini S and Maiorana A: Analysis of KRAS, NRAS, PIK3CA, and BRAF mutational profile in poorly differentiated clusters of KRAS-mutated colon cancer. Hum Pathol 62: 91-98, 2017.

8. Misale S, Yaeger R, Hobor S, Scala E, Janakiraman M, Liska D, Valtorta E, Schiavo R, Buscarino M, Siravegna G, et al: Emergence of KRAS mutations and acquired resistance to anti-EGFR therapy in colorectal cancer. Nature 486: 532-536, 2012.

9. Cooks T, Pateras IS, Jenkins LM, Patel KM, Robles AI, Morris J, Forshew T, Appella E, Gorgoulis VG and Harris CC: Mutant p53 cancers reprogram macrophages to tumor supporting macrophages via exosomal miR-1246. Nat Commun 9: 771, 2018.

10. Fu X, Huang Y, Fan X, Deng Y, Liu H, Zou H, Wu P, Chen Z, Huang J, Wang J, et al: Demographic trends and KRAS/BRAF ${ }^{\mathrm{V} 600 \mathrm{E}}$ mutations in colorectal cancer patients of South China: A single-site report. Int J Cancer 144: 2109-2117, 2019.

11. O'Brien MJ, Yang S, Mack C, Xu H, Huang CS, Mulcahy E, Amorosino $\mathrm{M}$ and Farraye FA: Comparison of microsatellite instability, $\mathrm{CpG}$ island methylation phenotype, BRAF and KRAS status in serrated polyps and traditional adenomas indicates separate pathways to distinct colorectal carcinoma end points. Am J Surg Pathol 30: 1491-1501, 2006.

12. Saito M, Momma T and Kono K: Targeted therapy according to next generation sequencing-based panel sequencing. Fukushima J Med Sci 64: 9-14, 2018.

13. Deshiere A, Berthet N, Lecouturier F, Gaudaire D and Hans A: Molecular characterization of Equine Infectious Anemia Viruses using targeted sequence enrichment and next generation sequencing. Virology 537: 121-129, 2019.

14. Meng H, Wang L, You H, Huang C and Li J: Circular RNA expression profile of liver tissues in an EtOH-induced mouse model of alcoholic hepatitis. Eur J Pharmacol 862: 172642, 2019.

15. Harada K, Okamoto W, Mimaki S, Kawamoto Y, Bando H, Yamashita R, Yuki S, Yoshino T, Komatsu Y, Ohtsu A, et al: Comparative sequence analysis of patient-matched primary colorectal cancer, metastatic, and recurrent metastatic tumors after adjuvant FOLFOX chemotherapy. BMC Cancer 19: 255, 2019.

16. Hu Y, He C, Liu JP, Li NS, Peng C, Yang-Ou YB, Yang XY, $\mathrm{Lu} \mathrm{NH}$ and Zhu Y: Analysis of key genes and signaling pathways involved in Helicobacter pylori-associated gastric cancer based on the cancer genome atlas database and RNA sequencing data. Helicobacter 23: e12530, 2018.

17. Nakagawa $\mathrm{H}$ and Fujita $\mathrm{M}$ : Whole genome sequencing analysis for cancer genomics and precision medicine. Cancer Sci 109: 513-522, 2018.

18. Barrett T, Wilhite SE, Ledoux P, Evangelista C, Kim IF, Tomashevsky M, Marshall KA, Phillippy KH, Sherman PM, Holko M, et al: NCBI GEO: Archive for functional genomics data sets-update. Nucleic Acids Res 41: D991-D995, 2013.

19. Huang DW, Sherman BT, Tan Q, Collins JR, Alvord WG, Roayaei J, Stephens R, Baseler MW, Lane HC and Lempicki RA: The DAVID gene functional classification tool: A novel biological module-centric algorithm to functionally analyze large gene lists. Genome Biol 8: R183, 2007.

20. Kanehisa M, Furumichi M, Tanabe M, Sato Y and Morishima K: KEGG: New perspectives on genomes, pathways, diseases and drugs. Nucleic Acids Res 45: D353-D361, 2017.

21. Ashburner M, Ball CA, Blake JA, Botstein D, Butler H, Cherry JM, Davis AP, Dolinski K, Dwight SS, Eppig JT, et al: Gene ontology: Tool for the unification of biology. The gene ontology consortium. Nat Genet 25: 25-29, 2000.

22. Franceschini A, Szklarczyk D, Frankild S, Kuhn M, Simonovic M, Roth A, Lin J, Minguez P, Bork P, von Mering C and Jensen LJ: STRING v9.1: Protein-protein interaction networks, with increased coverage and integration. Nucleic Acids Res 41: D808-D815, 2013. 
23. Smoot ME, Ono K, Ruscheinski J, Wang PL and Ideker T: Cytoscape 2.8: New features for data integration and network visualization. Bioinformatics 27: 431-432, 2011.

24. Bandettini WP, Kellman P, Mancini C, Booker OJ, Vasu S, Leung SW, Wilson JR, Shanbhag SM, Chen MY and Arai AE: MultiContrast Delayed Enhancement (MCODE) improves detection of subendocardial myocardial infarction by late gadolinium enhancement cardiovascular magnetic resonance: A clinical validation study. J Cardiovasc Magn Reson 14: 83, 2012.

25. Cerami E, Gao J, Dogrusoz U, Gross BE, Sumer SO, Aksoy BA Jacobsen A, Byrne CJ, Heuer ML, Larsson E, et al: The cBio cancer genomics portal: An open platform for exploring multidimensional cancer genomics data. Cancer Discov 2: 401-404, 2012.

26. Haeussler M, Zweig AS, Tyner C, Speir ML, Rosenbloom KR, Raney BJ, Lee CM, Lee BT, Hinrichs AS, Gonzalez JN, et al: The UCSC genome browser database: 2019 update. Nucleic Acids Res 47: D853-D858, 2019.

27. Rhodes DR, Yu J, Shanker K, Deshpande N, Varambally R, Ghosh D, Barrette T, Pandey A and Chinnaiyan AM: ONCOMINE: A cancer microarray database and integrated data-mining platform. Neoplasia 6: 1-6, 2004.

28. Wang ZZ, Yang J, Jiang BH, Di JB, Gao P, Peng L and Su XQ: KIF14 promotes cell proliferation via activation of Akt and is directly targeted by $\mathrm{miR}-200 \mathrm{c}$ in colorectal cancer. Int J Oncol 53: 1939-1952, 2018

29. Wu J, Yi J, Wu Y, Chen X, Zeng J, Wu J and Peng W: 3, 3'-dimethylquercetin inhibits the proliferation of human colon cancer RKO cells through Inducing G2/M cell cycle arrest and apoptosis. Anticancer Agents Med Chem 19: 402-409, 2019.

30. Cheng J, Dwyer M, Okolotowicz KJ, Mercola M and Cashman JR: A novel inhibitor targets both wnt signaling and ATM/p53 in colorectal cancer. Cancer Res 78: 5072-5083, 2018.

31. Li J, Liu YY, Yang XF, Shen DF, Sun HZ, Huang KQ and Zheng HC: Effects and mechanism of STAT3 silencing on the growth and apoptosis of colorectal cancer cells. Oncol Lett 16: 5575-5582, 2018

32. Chamberlain JA, Dugué PA, Bassett JK, Hodge AM, Brinkman MT, Joo JE, Jung CH, Makalic E, Schmidt DF, Hopper JL, et al: Dietary intake of one-carbon metabolism nutrients and DNA methylation in peripheral blood. Am J Clin Nutr 108: 611-621, 2018.

33. Ducker GS, Chen L, Morscher RJ, Ghergurovich JM, Esposito M, Teng X, Kang Y and Rabinowitz JD: Reversal of cytosolic one-carbon flux compensates for loss of the mitochondrial folate pathway. Cell Metab 23: 1140-1153, 2016.

34. Nitiss JL: DNA topoisomerase II and its growing repertoire of biological functions. Nat Rev Cancer 9: 327-337, 2009.

35. Pommier Y, Leo E, Zhang H and Marchand C: DNA topoisomerases and their poisoning by anticancer and antibacterial drugs. Chem Biol 17: 421-433, 2010

36. Deweese JE and Osheroff N: The DNA cleavage reaction of topoisomerase II: Wolf in sheep's clothing. Nucleic Acids Res 37: 738-748, 2009.

37. McLeod HL, Douglas F, Oates M, Symonds RP, Prakash D, van derZee AG, Kaye SB, Brown R and Keith WN: Topoisomerase I and II activity in human breast, cervix, lung and colon cancer. Int J Cancer 59: 607-611, 1994.

38. Shibao K, Takano H, Nakayama Y, Okazaki K, Nagata N, Izumi H, Uchiumi T, Kuwano M, Kohno K and Itoh H: Enhanced coexpression of YB-1 and DNA topoisomerase II alpha genes in human colorectal carcinomas. Int J Cancer 83: 732-737, 1999.

39. Coss A, Tosetto M, Fox EJ, Sapetto-Rebow B, Gorman S, Kennedy BN, Lloyd AT, Hyland JM, O'Donoghue DP, Sheahan K, et al: Increased topoisomerase IIalpha expression in colorectal cancer is associated with advanced disease and chemotherapeutic resistance via inhibition of apoptosis. Cancer Lett 276: 228-238, 2009.

40. Goswami MT, Chen G, Chakravarthi BV, Pathi SS, Anand SK, Carskadon SL, Giordano TJ, Chinnaiyan AM, Thomas DG Palanisamy N,et al: Role and regulation of coordinately expressed de novo purine biosynthetic enzymes PPAT and PAICS in lung cancer. Oncotarget 6: 23445-23461, 2015.

41. Meng M, Chen Y, Jia J, Li L and Yang S: Knockdown of PAICS inhibits malignant proliferation of human breast cancer cell lines. Biol Res 51: 24, 2018

42. Chakravarthi BVSK, Rodriguez Pena MDC, Agarwal S, Chandrashekar DS, Hodigere Balasubramanya SA, Jabboure FJ, Matoso A, Bivalacqua TJ, Rezaei K, Chaux A, et al: A role for de novo purine metabolic enzyme PAICS in bladder cancer progression. Neoplasia 20: 894-904, 2018.
43. Santamaría D, Barrière $\mathrm{C}$, Cerqueira $\mathrm{A}$, Hunt $\mathrm{S}$, Tardy $\mathrm{C}$, Newton K, Cáceres JF, Dubus P, Malumbres M and Barbacid M: Cdk1 is sufficient to drive the mammalian cell cycle. Nature 448: 811-815, 2007.

44. Brown NR, Korolchuk S, Martin MP, Stanley WA Moukhametzianov R, Noble MEM and Endicott JA: CDK1 structures reveal conserved and unique features of the essential cell cycle CDK. Nat Commun 6: 6769, 2015.

45. Asghar U, Witkiewicz AK, Turner NC and Knudsen ES: The history and future of targeting cyclin-dependent kinases in cancer therapy. Nat Rev Drug Discov 14: 130-146, 2015.

46. Goga A, Yang D, Tward AD, Morgan DO and Bishop JM: Inhibition of CDK1 as a potential therapy for tumors over-expressing MYC. Nat Med 13: 820-827, 2007.

47. Martinsson-Ahlzén HS, Liberal V, Grünenfelder B, Chaves SR Spruck CH and Reed SI: Cyclin-dependent kinase-associated proteins Cks1 and Cks2 are essential during early embryogenesis and for cell cycle progression in somatic cells. Mol Cell Biol 28: 5698-5709, 2008

48. Li M, Lin YM, Hasegawa S, Shimokawa T, Murata $\mathrm{K}$ Kameyama M, Ishikawa O, Katagiri T, Tsunoda T, Nakamura Y and Furukawa Y: Genes associated with liver metastasis of colon cancer, identified by genome-wide cDNA microarray. Int J Oncol 24: 305-312, 2004

49. Claesson-Welsh L and Welsh M: VEGFA and tumour angiogenesis. J Intern Med 273: 114-127, 2013.

50. Ferrara $\mathrm{N}$ and Adamis AP: Ten years of anti-vascular endothelial growth factor therapy. Nat Rev Drug Discov 15: 385-403, 2016.

51. Terme M, Pernot S, Marcheteau E, Sandoval F, Benhamouda N, Colussi O, Dubreuil O, Carpentier AF, Tartour E and Taieb J: VEGFA-VEGFR pathway blockade inhibits tumor-induced regulatory T-cell proliferation in colorectal cancer. Cancer Res 73: 539-549, 2013.

52. Seki A and Fang G: CKAP2 is a spindle-associated protein degraded by APC/C-Cdh1 during mitotic exit. J Biol Chem 282: 15103-15113, 2007.

53. Tsuchihara K, Lapin V, Bakal C, Okada H, Brown L, Hirota-Tsuchihara M, Zaugg K, Ho A, Itie-Youten A, Harris-Brandts M, et al: Ckap2 regulates aneuploidy, cell cycling, and cell death in a p53-dependent manner. Cancer Res 65: 6685-6691, 2005.

54. Weinberger P, Ponny SR, Xu H, Bai S, Smallridge R, Copland J and Sharma A: Cell cycle M-phase genes are highly upregulated in anaplastic thyroid carcinoma. Thyroid 27: 236-252,2017.

55. Wang Y, Jin T, Dai X and Xu J: Lentivirus-mediated knockdown of CEP55 suppresses cell proliferation of breast cancer cells. Biosci Trends 10: 67-73, 2016.

56. Jiang W, Wang Z, Chen G and Jia Y: Prognostic significance of centrosomal protein 55 in stage I pulmonary adenocarcinoma after radical resection. Thorac Cancer 7: 316-322, 2016.

57. D'Angiolella V, Donato V, Forrester FM, Jeong YT, Pellacani C, Kudo Y, Saraf A, Florens L, Washburn MP and Pagano M: Cyclin F-mediated degradation of ribonucleotide reductase $\mathrm{M} 2$ controls genome integrity and DNA repair. Cell 149: 1023-1034, 2012

58. Brognard $\mathrm{J}$ and Newton AC: PHLiPPing the switch on Akt and protein kinase $\mathrm{C}$ signaling. Trends Endocrinol Metab 19: 223-230, 2008

59. Brognard J, Sierecki E, Gao T and Newton AC: PHLPP and a second isoform, PHLPP2, differentially attenuate the amplitude of Akt signaling by regulating distinct Akt isoforms. Mol Cell 25: 917-931, 2007.

60. Liao WT, Li TT, Wang ZG, Wang SY, He MR, Ye YP, Qi L, Cui YM, Wu P, Jiao HL, et al: microRNA-224 promotes cell proliferation and tumor growth in human colorectal cancer by repressing PHLPP1 and PHLPP2. Clin Cancer Res 19: 4662-4672, 2013.

61. Cai J, Fang L, Huang Y, Li R, Yuan J, Yang Y, Zhu X, Chen B, Wu J and Li M: miR-205 targets PTEN and PHLPP2 to augment AKT signaling and drive malignant phenotypes in non-small cell lung cancer. Cancer Res 73: 5402-5415, 2013.

62. Santarpia L, Qi Y, Stemke-Hale K, Wang B, Young EJ, Booser DJ, Holmes FA, O'Shaughnessy J, Hellerstedt B, Pippen J, et al: Mutation profiling identifies numerous rare drug targets and distinct mutation patterns in different clinical subtypes of breast cancers. Breast Cancer Res Treat 134: 333-343, 2012.

63. Hu CM, Zhu J, Guo XE, Chen W, Qiu XL, Ngo B, Chien R, Wang YV, Tsai CY, Wu G, et al: Novel small molecules disrupting $\mathrm{Hec} 1 / \mathrm{Nek} 2$ interaction ablate tumor progression by triggering Nek2 degradation through a death-trap mechanism. Oncogene 34: 1220-1230, 2015. 
64. Hayward DG, Clarke RB, Faragher AJ, Pillai MR, Hagan IM and Fry AM: The centrosomal kinase Nek2 displays elevated levels of protein expression in human breast cancer. Cancer Res 64: 7370-7376, 2004.

65. Hawkins SM, Loomans HA, Wan YW, Ghosh-Choudhury T Coffey D, Xiao W, Liu Z, Sangi-Haghpeykar H and Anderson ML: Expression and functional pathway analysis of nuclear receptor NR2F2 in ovarian cancer. J Clin Endocrinol Metab 98: E1152-E1162, 2013.

66. Neal CP, Fry AM, Moreman C, McGregor A, Garcea G, Berry DP and Manson MM: Overexpression of the Nek 2 kinase in colorectal cancer correlates with beta-catenin relocalization and shortened cancer-specific survival. J Surg Oncol 110: 828-838, 2014.

67. Lu L, Zhai X and Yuan R: Clinical significance and prognostic value of Nek2 protein expression in colon cancer. Int J Clin Exp Pathol 8: 15467-15473, 2015.
68. Xu H, Zeng L, Guan Y, Feng X, Zhu Y, Lu Y, Shi C, Chen S, $\mathrm{Xia} J$, Guo J, et al: High NEK2 confers to poor prognosis and contributes to cisplatin-based chemotherapy resistance in nasopharyngeal carcinoma. J Cell Biochem 120: 3547-3558, 2019.

69. Zhang Y, Wang W, Wang Y, Huang X, Zhang Z, Chen B, Xie W, Li S, Shen S and Peng B: NEK2 promotes hepatocellular carcinoma migration and invasion through modulation of the epithelial-mesenchymal transition. Oncol Rep 39: 1023-1033, 2018.

(i) This work is licensed under a Creative Commons Attribution-NonCommercial-NoDerivatives 4.0 International (CC BY-NC-ND 4.0) License. 